\title{
BMJ Open Associations of obesity, physical activity level, inflammation and cardiometabolic health with COVID-19 mortality: a prospective analysis of the UK Biobank cohort
}

\author{
Malik Hamrouni (D), Matthew J Roberts, Alice Thackray, David J Stensel (D), \\ Nicolette Bishop
}

To cite: Hamrouni M, Roberts MJ, Thackray A, et al. Associations of obesity, physical activity level, inflammation and cardiometabolic health with COVID-19 mortality: a prospective analysis of the UK Biobank cohort. BMJ Open 2021;11:e055003. doi:10.1136/ bmjopen-2021-055003

- Prepublication history and additional supplemental material for this paper are available online. To view these files, please visit the journal online (http://dx.doi.org/10.1136/ bmjopen-2021-055003).

Received 30 June 2021 Accepted 22 0ctober 2021

D Check for updates

(c) Author(s) (or their employer(s)) 2021. Re-use permitted under CC BY-NC. No commercial re-use. See rights and permissions. Published by BMJ.

School of Sport, Exercise and Health Sciences, Loughborough University, Loughborough, UK

Correspondence to Professor Nicolette Bishop; N.C.Bishop@lboro.ac.uk

\section{ABSTRACT}

Objectives To investigate the associations of physical activity level with COVID-19 mortality risk across body mass index (BMI) categories, and to determine whether any protective association of a higher physical activity level in individuals with obesity may be explained by favourable levels of cardiometabolic and inflammatory biomarkers. Design Prospective cohort study (baseline data collected between 2006 and 2010). Physical activity level was assessed using the International Physical Activity Questionnaire (high: $\geq 3000$ Metabolic Equivalent of Task (MET)-min/week, moderate: $\geq 600$ MET-min/ week, low: not meeting either criteria), and biochemical assays were conducted on blood samples to provide biomarker data.

Setting UK Biobank.

Main outcome measures Logistic regressions adjusted for potential confounders were performed to determine the associations of exposure variables with COVID-19 mortality risk. Mortality from COVID-19 was ascertained by death certificates through linkage with National Health Service (NHS) Digital.

Results Within the 259397 included participants, 397 COVID-19 deaths occurred between 16 March 2020 and 27 February 2021. Compared with highly active individuals with a normal BMI (reference group), the 0Rs $(95 \% \mathrm{Cls}$ ) for COVID-19 mortality were 1.61 (0.98 to 2.64) for highly active individuals with obesity, 2.85 (1.78 to 4.57 ) for lowly active individuals with obesity and 1.94 (1.04 to 3.61) for lowly active individuals with a normal BMI. Of the included biomarkers, neutrophil count and monocyte count were significantly positively associated with COVID-19 mortality risk. In a subanalysis restricted to individuals with obesity, adjusting for these biomarkers attenuated the higher COVID-19 mortality risk in lowly versus highly active individuals with obesity by $10 \%$.

Conclusions This study provides novel evidence suggesting that a high physical activity level may attenuate the COVID-19 mortality risk associated with obesity. Although the protective association may be partly explained by lower neutrophil and monocyte counts, it still remains largely unexplained by the biomarkers included in this analysis.
Strengths and limitations of this study

- Comprehensive individual data within the UK Biobank allowed adjustment for several confounding variables.

- Baseline assessment was conducted at least 10 years before the COVID-19 pandemic which rules out reverse causation (eg, severe COVID-19 causing a reduction in physical activity), a particular concern in prognostic studies of patient populations.

- Physical activity level was assessed by self-report, which is susceptible to bias due to subjectivity.

- UK Biobank sample is not representative of the general population (participants are less socioeconomically deprived and have fewer health conditions).

- All analysed risk factors were subject to change between baseline data collection and the COVID-19 pandemic.

\section{INTRODUCTION}

The consequences of exposure to SARSCoV-2, which causes COVID-19, range from asymptomatic disease to critical health conditions that can result in hospitalisation and death. ${ }^{1}$ Obesity has been identified as a major risk factor for both disease severity and mortality from COVID-19. ${ }^{2}$ Higher levels of systemic inflammation and impaired cardiovascular and metabolic health are suggested to be key features that predispose the greater risk of COVID-19 morbimortality in individuals with obesity. ${ }^{3}$

Regular physical activity can exhibit antiinflammatory effects and is associated with better immune defence and cardiometabolic health. ${ }^{45}$ Consequently, physical activity has been proposed as an important protective behaviour against adverse COVID-19 health outcomes. ${ }^{67}$ In support of this notion, physically active individuals have recently been 
shown to be at lower risk of COVID-19 mortality. ${ }^{8}$ However, no studies have investigated whether a protective association of physical activity against COVID-19 mortality may also be apparent in obesity. Physically active individuals with obesity exhibit better cardiometabolic health and lower systemic inflammation compared with their less active counterparts. ${ }^{9-11}$ Importantly, this suggests the potential for higher levels of physical activity to reduce the burden of COVID-19 typically associated with obesity.

Although the COVID-19 vaccination programme is underway in the UK, worldwide delivery will present challenges and effects will not be instantaneous. ${ }^{12}$ In turn, it is imperative to continue to develop our understanding of how modifiable lifestyle factors may influence the burden of COVID-19, particularly in at-risk populations. Therefore, using the UK Biobank, this study will investigate the associations of body mass index (BMI) category, physical activity level, and cardiometabolic and inflammatory biomarkers with COVID-19 mortality risk. Further, we aim to determine if any protective association of a higher physical activity level may extend to individuals with obesity, and whether this may be explained by favourable biomarker profiles.

\section{METHODS}

All data were provided by UK Biobank, a prospective cohort in the UK that collected health-related measures from over 500000 participants aged 37-73 years, between 2006 and 2010. ${ }^{13}$ In the present study, we used data collected at baseline from UK Biobank assessment centres as exposures, in conjunction with COVID-19 mortality data, available through linkage of the cohort with National Health Service (NHS) Digital. The analysis conducted for this study covers the period between 16 March 2020 and 27 February 2021.

\section{Participant demographics}

Age at baseline was derived from date of birth and date of attending assessment centre. Current age was estimated as age on 1 March 2020. Height and body mass were measured at baseline using a Seca $240 \mathrm{~cm}$ height measure and a Tanita BC418MA body composition analyser, respectively. BMI was calculated from body mass $(\mathrm{kg})$ divided by height squared $\left(\mathrm{m}^{2}\right)$ and was categorised into three groups (normal 18.5-24.9 $\mathrm{kg} / \mathrm{m}^{2}$, overweight 25.0-29.9 $\mathrm{kg} / \mathrm{m}^{2}$, obesity $30 \mathrm{~kg} / \mathrm{m}^{2}$ or above). Individuals who were underweight $(\mathrm{n}=1255)$ were not included in the analysis due to limited COVID-19 deaths $(n=2)$. Sex was acquired from NHS records at recruitment. Additional information on potential confounders at the baseline assessment was assessed via self-report using a touchscreen questionnaire, including ethnicity (white, black, South Asian or mixed/other), smoking status (current, never, previous), alcohol intake frequency (daily/almost daily, one to two times/week, one to three times/month, special occasions only, never) and highest level of educational attainment (college/university degree, A-levels/AS-levels or equivalent, GCSEs or equivalent, none of the above). The number of illnesses (cancer and non-cancer illnesses) and number of treatments or medications for each participant at baseline were also assessed by touchscreen questionnaire. Townsend Deprivation Index, whereby higher values imply a greater degree of deprivation, was calculated for each participant using national census output for their postcode at the time of recruitment. ${ }^{14}$

\section{Comorbidities}

Comorbidities were assessed at baseline and updated over the follow-up period using participant linkage with hospital episode statistics (occurrences of specific International Classification of Diseases 10th revision (ICD-10) codes in hospital admission data up until 27 February 2021). Listed comorbidities include cardiovascular diseases (ischaemic heart disease, hypertensive disease, pulmonary heart disease and cerebrovascular disease), respiratory diseases (asthma, chronic obstructive pulmonary disease, chronic bronchitis, emphysema and bronchiectasis), diabetes mellitus, liver disease, kidney disease and cancer.

\section{Physical activity level}

Physical activity level was assessed using the short-form International Physical Activity Questionnaire (IPAQ), which classifies individuals into either 'low', 'moderate' or 'high', based on Metabolic Equivalent of Task (MET) minutes per week for walking and moderate-vigorous intensity physical activity. ${ }^{15}$ High and moderate physical activity levels were classed as achieving $\geq 3000$ and $\geq 600$ MET-min per week of any combination of walking, moderate-intensity or vigorous-intensity activity, respectively. Individuals who did not meet such criteria were considered to have a low physical activity level. More detailed information on the thresholds used to categorise different physical activity levels can be found in the data processing guidelines developed by IPAQ ${ }^{16}$

\section{Inflammatory and cardiometabolic biomarkers}

Non-fasting venous blood samples were collected at the baseline assessment and a wide range of biochemical assays were conducted to provide individual data on biomarkers that are established risk factors for disease and/or valuable clinical diagnostic measures. Detailed procedures for UK Biobank blood sample collection and processing are provided in the UK Biobank manual on blood sample collection and processing. ${ }^{17}$ The present study included $\mathrm{C}$ reactive protein (CRP) and total and differential leucocyte counts (neutrophil, lymphocyte and monocyte count) as markers of systemic inflammation, and total cholesterol, high-density lipoprotein cholesterol (HDL-C), low-density lipoprotein cholesterol (LDL$\mathrm{C})$, triglycerides (TG), glucose and glycated haemoglobin (HbA1c) as markers of cardiometabolic health.

\section{COVID-19 mortality data}

COVID-19 mortality was determined from the presence of ICD-10 codes U07.1 (virus identified in laboratory 
testing) or U07.2 (clinical or epidemiological diagnosis) as the primary cause on the death certificate.

\section{Statistical analysis}

Participant demographics were presented as median and IQR for continuous and discrete variables (due to non-normal distribution) and number and percentage for categorical variables. Comorbidities were presented as number and percentage. Separate logistic regressions were carried out to identify the associations of BMI category, physical activity level and cardiometabolic and inflammatory biomarkers with COVID-19 mortality. Next, we assessed the risk of COVID-19 mortality for the different combinations of physical activity level (high, moderate, low) and BMI category (normal, overweight, obesity), with highly active individuals with a normal BMI as the reference group.

To determine whether a potential protective association of a higher physical activity level against COVID-19 mortality in individuals with obesity may be explained by favourable biomarker profiles, we performed additional logistic regressions in a subanalysis restricted to individuals with obesity stratified by physical activity level. These logistic regressions were performed in two models, with model 1 adjusted for potential confounders and model 2 further adjusted for cardiometabolic and/ or inflammatory biomarkers found to be associated with COVID-19 mortality. The degree to which cardiometabolic and/or inflammatory biomarkers may explain the association between physical activity level and COVID-19 mortality in individuals with obesity was ascertained from the percentage change in the effect estimate upon their inclusion in the biomarker-adjusted model, in line with previous studies. ${ }^{18} 19$ Percentage change was calculated from the change in value divided by the original value, multiplied by 100 (using ORs expressed as percentages). This analysis was conducted in individuals with obesity as opposed to across all BMI categories, to ensure that adjustment was assessing factors that may contribute to the effect of physical activity level and not differences in BMI category.

As there may be similar risk factors for severe and fatal COVID-19, sensitivity analysis was conducted to determine whether exclusion of individuals with a positive SARSCoV-2 test result from an inpatient setting ${ }^{20}$ who did not die from COVID-19 altered the results. Another reason for conducting sensitivity analysis is that as COVID-19 testing data are updated more frequently than mortality data, it is possible that a lag may exist between inpatients with COVID-19 who die from the infection and this occurrence appearing in the mortality records. Analysis for this study was therefore restricted to participants with measurements taken at assessment centres in England (since dynamic linkage of COVID-19 testing results are exclusively provided by Public Health England). ${ }^{20}$

Potential confounders included baseline age, sex, ethnicity, BMI, physical activity level, smoking status, alcohol intake frequency, educational attainment,
Main analysis $(n=259$ 397)
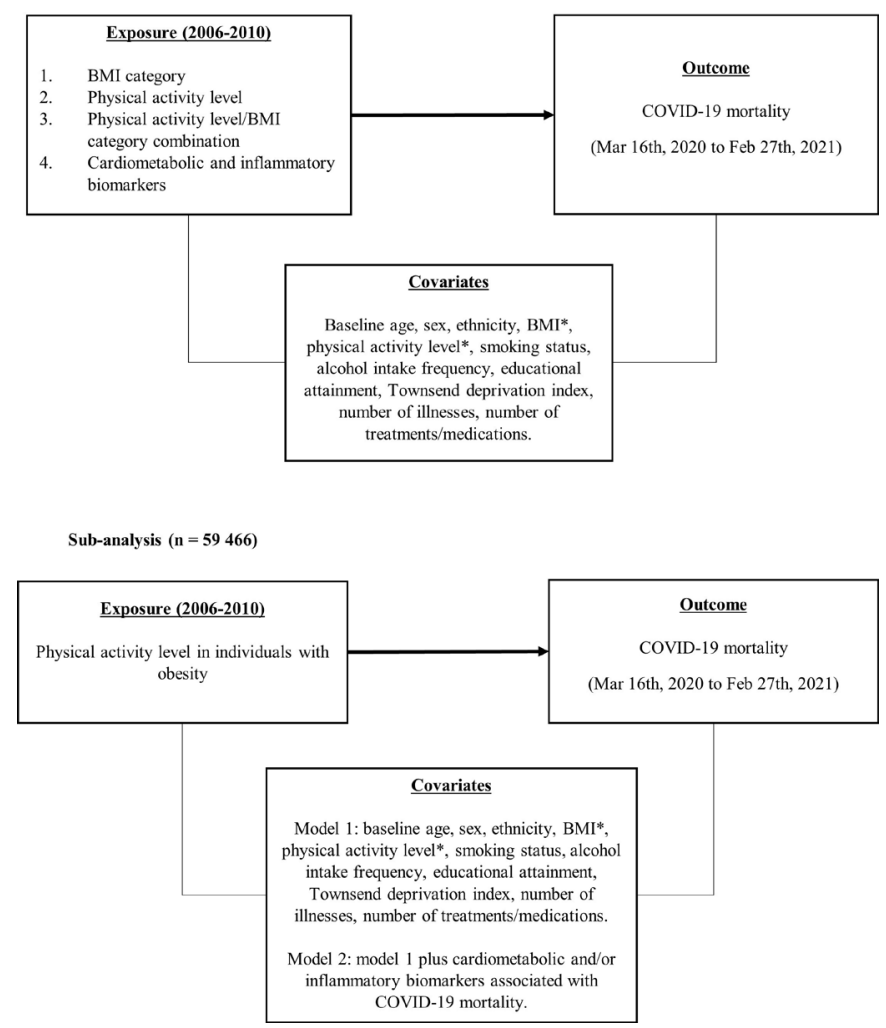

Figure 1 Path diagram for main analysis and subanalysis. *Variable not adjusted for when it is the exposure.

BMI, body mass index.

Townsend Deprivation Index, number of illnesses, and number of treatments or medications taken. Logistic regressions were adjusted for all potential confounders, except for BMI when stratifying participants by BMI category and physical activity level when stratifying participants by physical activity level. Results from logistic regressions were reported as ORs with 95\% CIs. Statistical significance was accepted at the alpha level of 0.05 , and the corresponding $95 \%$ CIs were calculated. All statistical analyses were performed with Stata V.16 (StataCorp, College Station, Texas, USA). A path diagram to illustrate the study design is presented in figure 1.

\section{Patient and public involvement}

No participants contributed to setting the research questions or determining the study design. Direct dissemination of the results to participants is not possible/ applicable.

\section{RESULTS}

The analytical sample was composed of 259397 participants from English assessment centres after excluding those who were lost to follow-up, those who died before 16 March 2020, those with missing data and underweight individuals (online supplemental figure 1). Within this sample, there were 397 COVID-19 deaths between 16 March 2020 and 27 February 2021. Participant 
demographics are presented for individuals who did and did not die from COVID-19 in table 1. Participants who died from COVID-19 were more likely to be older, male, current or former smokers, as well as have obesity, a low physical activity level and lower educational attainment. Additionally, the Townsend Deprivation Index, the number of illnesses, and the number of treatments and medications taken were higher for participants who died from COVID-19. Regarding comorbidities, individuals who died from COVID-19 had a higher prevalence of the listed cardiovascular and respiratory diseases, as well as diabetes, liver disease, kidney disease and cancer (table 2).

\section{Associations of BMI category with COVID-19 mortality independent of physical activity level}

Compared with those with a normal BMI (reference group), the risk of COVID-19 mortality was significantly higher for individuals with overweight and obesity (table 3).

\section{Associations of physical activity level with COVID-19 mortality} independent of BMI

Individuals with a low physical activity level were at a significantly higher risk of COVID-19 mortality compared with highly active individuals (reference group), whereas moderately active individuals were not at a significantly higher risk (table 4).

\section{Associations of physical activity level with COVID-19 mortality} across BMI categories

The associations of physical activity level with COVID-19 mortality across BMI categories are presented in figure 2. A low physical activity level was associated with a significantly higher COVID-19 mortality risk for individuals with a normal BMI (adjusted $\mathrm{OR}=1.94,95 \% \mathrm{CI}=1.04$ to 3.61 ), overweight $(1.70,1.03$ to 2.83$)$ and obesity $(2.85,1.78$ to 4.57 ), compared with highly active individuals with a normal BMI (reference group). Moderately active individuals with obesity were still at significantly greater risk $(1.96,1.23$ to 3.13$)$, whereas moderately active individuals with a normal BMI were not at higher risk $(0.93,0.53$ to $1.63)$, compared with the reference group. While moderately active individuals with overweight (1.52, 0.97 to 2.38$)$ were at higher risk compared with the reference group, this did not reach statistical significance. The COVID-19 mortality risk was still higher in highly active individuals with obesity $(1.61,0.98$ to 2.64$)$ and overweight $(1.51,0.96$ to 2.35) compared with the reference group; however, these associations were also non-significant.

Associations of cardiometabolic and inflammatory biomarkers with COVID-19 mortality

Total leucocyte, neutrophil and monocyte count were positively associated with COVID-19 mortality risk, although CRP and lymphocyte count did not exhibit a significant association (table 5). There was no significant association for glucose, HbA1c, total cholesterol, LDL-C, HDL-C and TG with COVID-19 mortality (table 5).
Table 1 Participant demographics for individuals who did and did not die from COVID-19

\begin{tabular}{|c|c|c|}
\hline Variables & $\begin{array}{l}\text { No COVID-19 } \\
\text { mortality } \\
(\mathrm{N}=259 \text { 000) }\end{array}$ & $\begin{array}{l}\text { COVID-19 } \\
\text { mortality } \\
(\mathrm{N}=397)\end{array}$ \\
\hline Baseline age (years) & $57(49-63)$ & $65(61-67)$ \\
\hline Current age (years) ${ }^{\star}$ & $68(61-74)$ & $76(72-78)$ \\
\hline \multicolumn{3}{|l|}{ Sex } \\
\hline Female & $135763(52 \%)$ & $121(30 \%)$ \\
\hline Male & $123237(48 \%)$ & $276(70 \%)$ \\
\hline \multicolumn{3}{|l|}{ Ethnicity } \\
\hline White & 246065 (95\%) & $364(92 \%)$ \\
\hline South Asian & $4843(2 \%)$ & $11(3 \%)$ \\
\hline Black & $3518(1 \%)$ & $16(4 \%)$ \\
\hline Mixed/other & $4574(2 \%)$ & $6(2 \%)$ \\
\hline \multicolumn{3}{|l|}{ Smoking status } \\
\hline Never & $143497(55 \%)$ & $144(36 \%)$ \\
\hline Previous & $90730(35 \%)$ & $194(49 \%)$ \\
\hline Current & $24773(10 \%)$ & $59(15 \%)$ \\
\hline \multicolumn{3}{|c|}{ Alcohol intake frequency } \\
\hline Daily/almost daily & $55736(22 \%)$ & $86(22 \%)$ \\
\hline 3-4 times/week & $62780(24 \%)$ & $67(17 \%)$ \\
\hline 1-2 times/week & $66766(26 \%)$ & $96(24 \%)$ \\
\hline 1-3 times/month & $28474(11 \%)$ & $36(9 \%)$ \\
\hline Special occasions & $27013(10 \%)$ & $60(15 \%)$ \\
\hline Never & $18231(7 \%)$ & $52(13 \%)$ \\
\hline
\end{tabular}

Educational

attainment

\begin{tabular}{lll}
$\begin{array}{l}\text { University or } \\
\text { college degree }\end{array}$ & $90618(35 \%)$ & $70(18 \%)$ \\
$\begin{array}{l}\text { A/AS-levels or } \\
\text { equivalent }\end{array}$ & $30769(12 \%)$ & $31(8 \%)$ \\
$\begin{array}{l}\text { GCSEs or } \\
\text { equivalent }\end{array}$ & $71214(28 \%)$ & $88(21 \%)$ \\
\hline $\begin{array}{l}\text { None of the } \\
\text { above }\end{array}$ & $66399(26 \%)$ & $208(53 \%)$ \\
\hline $\begin{array}{l}\left.\text { BMI (kg/m }{ }^{2}\right) \\
\text { BMl category }\end{array}$ & $26.6(24.1-29.7)$ & $28.8(26.0-32.8)$ \\
\hline $\begin{array}{l}\text { Normal } \\
\text { Overweight }\end{array}$ & $111939(43 \%)$ & $176(44 \%)$ \\
\hline $\begin{array}{l}\text { Obesity } \\
\text { Physical activity } \\
\text { level }\end{array}$ & $59310(23 \%)$ & $156(39 \%)$ \\
\hline $\begin{array}{l}\text { Low } \\
\text { Moderate }\end{array}$ & $105414(41 \%)$ & $150(38 \%)$ \\
\hline $\begin{array}{l}\text { High } \\
\text { Number of } \\
\text { illnesses }\end{array}$ & $105868(41 \%)$ & $138(34 \%)$ \\
\hline & $1(1-3)$ & $3(1-4)$ \\
\hline
\end{tabular}

Continued 
Table 1 Continued

\begin{tabular}{lll}
\hline Variables & $\begin{array}{l}\text { No COVID-19 } \\
\text { mortality } \\
\text { (N=259 000) }\end{array}$ & $\begin{array}{l}\text { COVID-19 } \\
\text { mortality } \\
\text { (N=397) }\end{array}$ \\
\hline $\begin{array}{l}\text { Number of } \\
\text { treatments/ } \\
\text { medications }\end{array}$ & $2(0-3)$ & $4(2-6)$ \\
\hline Townsend Index† & $-2.3(-3.7$ to 0.2$)$ & $-1.2(-3.1$ to 2.1$)$ \\
\hline
\end{tabular}

Characteristics presented as median (25th-75th percentiles) for continuous and discrete variables, and number (percentage) for categorical variables.

*Age as of 1 March 2020.

†Higher values for Townsend Deprivation Index imply a greater degree of deprivation.

BMl, body mass index.

Biomarker-adjusted associations of physical activity level with COVID-19 mortality in individuals with obesity

The biomarker-adjusted associations of physical activity level with COVID-19 mortality in individuals with obesity can be found in table 6 . In model 1, compared with highly active individuals with obesity (reference group), moderately active individuals with obesity were not at significantly higher risk of COVID-19 mortality, but lowly active individuals with obesity were (1.80, 1.19 to 2.72). However, in model 2, adjusting for the biomarkers associated with COVID-19 mortality (neutrophil and monocyte count) attenuated this effect estimate by $10 \%(1.72,1.15$ to 2.61 ).

Table 2 Comorbidities in individuals who did and did not die from COVID-19

\begin{tabular}{|lll} 
Comorbidity & $\begin{array}{l}\text { No COVID-19 } \\
\text { mortality } \\
\text { (N=259 000) }\end{array}$ & $\begin{array}{l}\text { COVID-19 } \\
\text { mortality } \\
\text { (N=397) }\end{array}$ \\
\hline $\begin{array}{l}\text { Cardiovascular diseases } \\
\text { Ischaemic heart disease }\end{array}$ & $26387(10 \%)$ & $125(31 \%)$ \\
Hypertensive disease & $73480(28 \%)$ & $285(72 \%)$ \\
\hline Pulmonary heart disease & $4054(2 \%)$ & $49(12 \%)$ \\
\hline Cerebrovascular disease & $8585(3 \%)$ & $98(25 \%)$ \\
Respiratory diseases & & \\
\hline Asthma & $23231(9 \%)$ & $58(15 \%)$ \\
\hline COPD & $8141(3 \%)$ & $84(21 \%)$ \\
\hline Chronic bronchitis & $859(<1 \%)$ & $5(1 \%)$ \\
\hline Emphysema & $1665(1 \%)$ & $28(7 \%)$ \\
\hline Bronchiectasis & $2386(1 \%)$ & $18(5 \%)$ \\
Diabetes mellitus & $18982(7 \%)$ & $128(32 \%)$ \\
Liver disease & $5981(2 \%)$ & $51(13 \%)$ \\
Kidney disease & $8930(3 \%)$ & $94(24 \%)$ \\
Cancer (malignant neoplasm) & $39993(15 \%)$ & $118(30 \%)$ \\
\hline
\end{tabular}

Comorbidities are presented as number (percentage).

COPD, chronic obstructive pulmonary disease.
Table 3 Associations of BMI category with COVID-19 mortality

\begin{tabular}{lcl}
\hline BMI category & N deaths/N total & $\begin{array}{l}\text { COVID-19 mortality } \\
\text { OR }(95 \% \text { Cl) }\end{array}$ \\
\hline Normal & $65 / 87816$ & 1 (reference) \\
Overweight & $176 / 112115$ & $\mathbf{1 . 3 8}(\mathbf{1 . 0 3}$ to 1.84$)$ \\
Obesity & $156 / 59466$ & $\mathbf{1 . 7 9}(\mathbf{1 . 3 2}$ to 2.43$)$
\end{tabular}

Adjusted for baseline age, sex, ethnicity, physical activity level, Townsend Deprivation Index, smoking status, alcohol intake, educational attainment, number of illnesses and number of treatments/medications taken.

Significant associations $(p<0.05)$ are highlighted in bold. BMI, body mass index.

\section{Sensitivity analysis}

Removal of inpatients who tested positive for SARS-CoV-2 who did not die from COVID-19 $(n=1719)$ from the analysis resulted in similar findings to the main analysis (online supplemental tables 1-5).

\section{DISCUSSION}

A compelling finding from our analysis of the UK Biobank cohort was that a high pre-pandemic physical activity level attenuated the COVID-19 mortality risk associated with obesity. Furthermore, our analysis of biomarkers revealed that higher pre-pandemic total leucocyte, neutrophil and monocyte counts were associated with a higher COVID-19 mortality risk. Interestingly, the biomarker-adjusted model restricted to individuals with obesity indicated that the lower COVID-19 mortality risk in highly versus lowly active individuals with obesity may be partly explained by lower neutrophil and monocyte counts. However, the protective association for a high pre-pandemic physical activity level in obesity still remains largely unexplained by the biomarkers included in the present study.

In accordance with Sallis $e t a l^{8}{ }^{8}$ we found that a low pre-pandemic physical activity level was a risk factor for COVID-19 mortality independent of BMI. A principal novel finding in the present study, however, is that a high pre-pandemic physical activity level attenuated the

Table 4 Associations of physical activity level with COVID-19 mortality

\begin{tabular}{lll}
$\begin{array}{l}\text { Physical activity } \\
\text { level }\end{array}$ & N deaths/N total & $\begin{array}{l}\text { COVID-19 mortality } \\
\text { OR }(95 \% \text { Cl) }\end{array}$ \\
\hline High & $138 / 106006$ & 1 (reference) \\
Moderate & $150 / 105564$ & $1.05(0.83$ to 1.33$)$ \\
Low & $109 / 47827$ & $\mathbf{1 . 4 1}(\mathbf{1 . 0 9}$ to 1.83$)$ \\
\hline
\end{tabular}

Adjusted for baseline age, sex, ethnicity, BMI, Townsend Deprivation Index, smoking status, alcohol intake, educational attainment, number of illnesses and number of treatments/ medications taken.

Significant associations $(p<0.05)$ are highlighted in bold. BMI, body mass index. 
Physical activity level and COVID-19 mortality across BMI categories

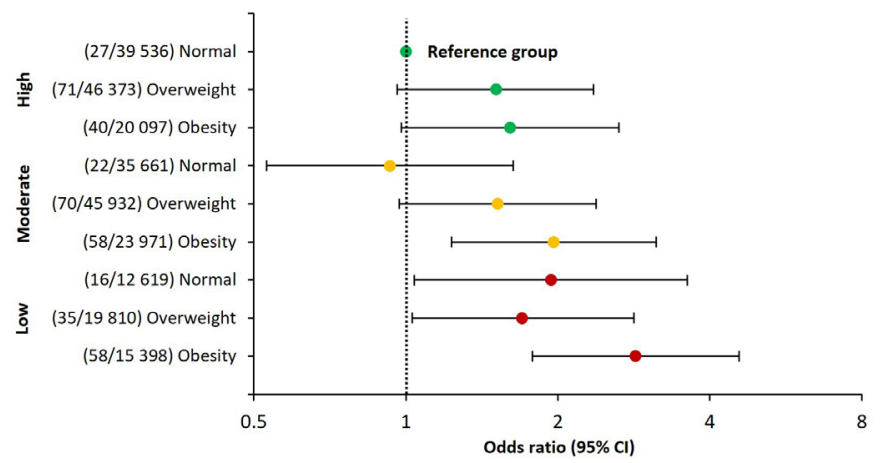

Figure 2 Associations of physical activity level with COVID-19 mortality across BMI categories. Adjusted for baseline age, sex, ethnicity, Townsend Deprivation Index, smoking status, alcohol intake, educational attainment, number of illnesses, and number of treatments and/or medications taken. Numbers in brackets are COVID-19 deaths/total sample population.

BMI, body mass index.

elevated risk of COVID-19 mortality in individuals with obesity. This finding is consistent with research indicating that physical activity has a protective effect on the hazards typically associated with obesity, such as elevated all-cause mortality and cardiometabolic disease risk. ${ }^{21}$ Our results suggest there may have been a disproportionate amount of attention drawn to obesity compared with physical inactivity as a risk factor during the pandemic. Indeed, we found that lowly active individuals with a normal BMI

Table 5 Associations of cardiometabolic and inflammatory biomarkers with COVID-19 mortality

\begin{tabular}{ll}
\hline Biomarker & $\begin{array}{l}\text { COVID-19 mortality } \\
\text { OR }(95 \% \text { Cl) }\end{array}$ \\
\hline CRP $(\mathrm{mg} / \mathrm{L})$ & $1.01(0.99$ to 1.03$)$ \\
\hline Total leucocyte count $\left(10^{9} / \mathrm{L}\right)$ & $\mathbf{1 . 0 3}(\mathbf{1 . 0 1}$ to 1.05$)$ \\
\hline Neutrophil count $\left(10^{9} / \mathrm{L}\right)$ & $\mathbf{1 . 1 0}(\mathbf{1 . 0 3}$ to 1.18$)$ \\
\hline Lymphocyte count $\left(10^{9} / \mathrm{L}\right)$ & $1.02(0.98$ to 1.07$)$ \\
\hline Monocyte count $\left(10^{9} / \mathrm{L}\right)$ & $\mathbf{1 . 3 2}(\mathbf{1 . 0 3}$ to 1.68$)$ \\
\hline Total cholesterol $(\mathrm{mmol} / \mathrm{L})$ & $0.98(0.89$ to 1.07$)$ \\
LDL-C (mmol/L) & $0.98(0.87$ to 1.11$)$ \\
\hline HDL-C $(\mathrm{mmol} / \mathrm{L})$ & $0.99(0.70$ to 1.38$)$ \\
\hline TG $(\mathrm{mmol} / \mathrm{L})$ & $1.01(0.92$ to 1.11$)$ \\
\hline Glucose $(\mathrm{mmol} / \mathrm{L})$ & $1.05(1.00$ to 1.11$)$ \\
\hline HbA1c $(\mathrm{mmol} / \mathrm{L})$ & $1.01(1.00$ to 1.02$)$ \\
\hline
\end{tabular}

OR (95\% Cls) for COVID-19 mortality per one unit increase in each biomarker. Adjusted for baseline age, sex, ethnicity, BMI, physical activity level, Townsend Deprivation Index, smoking status, alcohol intake, educational attainment, number of illnesses and number of treatments/medications taken.

Significant associations $(p<0.05)$ are highlighted in bold.

$\mathrm{BMI}$, body mass index; CRP, C reactive protein; $\mathrm{HbA1c}$, glycated haemoglobin; HDL-C, high-density lipoprotein cholesterol; LDL-C, low-density lipoprotein cholesterol; TG, triglycerides. were at a significantly higher risk of COVID-19 mortality compared with highly active individuals with a normal BMI (reference group), whereas highly active individuals with obesity were not. The observed potential for higher levels of physical activity to mitigate the risk of COVID-19 mortality in obesity is crucial considering the low success rate of weight loss-focused interventions. ${ }^{22}$ Although speculative, encouraging inactive individuals with obesity to partake in regular physical activity may be a more realistic strategy than weight loss-focused interventions to protect against mortality from COVID-19.

However, a high physical activity level may not completely negate the higher COVID-19 mortality risk associated with obesity. Although the COVID-19 mortality risk in highly active individuals with obesity was not significantly higher compared with highly active individuals with a normal BMI, the magnitude of the OR (1.61) and the lower 95\% CI approaching 1.00 (0.98) suggests that the former may still be at elevated risk of COVID-19 mortality. Therefore, weight loss may still be necessary to further lower the COVID-19 mortality risk in highly active individuals with obesity.

Although one UK Biobank study suggested that elevated pre-pandemic total leucocyte, neutrophil and monocyte counts may be linked with greater risk of severe COVID$19,{ }^{23}$ to our knowledge, this is the first study demonstrating an association for higher risk of COVID-19 mortality. Moreover, in the analysis restricted to individuals with obesity, biomarker adjustment revealed that higher neutrophil and monocyte counts may partly explain the elevated COVID-19 mortality risk observed in individuals with obesity who had low levels of physical activity compared with their highly active counterparts. An excessive inflammatory response to SARS-CoV-2 infection, termed 'cytokine storm', has been identified as a major cause of death in patients with COVID$19 .^{24}$ One of the key pathological features of the cytokine storm is an abundance of neutrophils and monocytes infiltrating alveolar spaces in response to upregulated proinflammatory cytokine and chemokine release. ${ }^{25-27}$ Regular physical activity is associated with lower total and differential leucocyte counts. ${ }^{28-30}$ Therefore, to explain our findings, we speculate that higher premorbid neutrophil and monocyte counts in physically inactive individuals with obesity may result in a greater number of such cells migrating from the circulation into alveolar spaces during infection. In turn, this may contribute to increased disease severity and thus partly explain the greater COVID-19 mortality risk observed in lowly versus highly active individuals with obesity.

The protective association of a high pre-pandemic physical activity level in individuals with obesity still remains largely unexplained by the biomarkers included in the present study. However, this does not refute the notion that higher levels of physical activity in obesity may confer protection against COVID-19 mortality largely through beneficial effects on inflammation and cardiometabolic health, as there may be other related mechanisms that contribute to explaining our findings. Indeed, looking at the circulating levels of a relatively small selection of traditional biomarkers 
Table 6 Effect of biomarker adjustment on the associations of physical activity level with COVID-19 mortality in individuals with obesity

COVID-19 mortality OR $(95 \% \mathrm{Cl})$

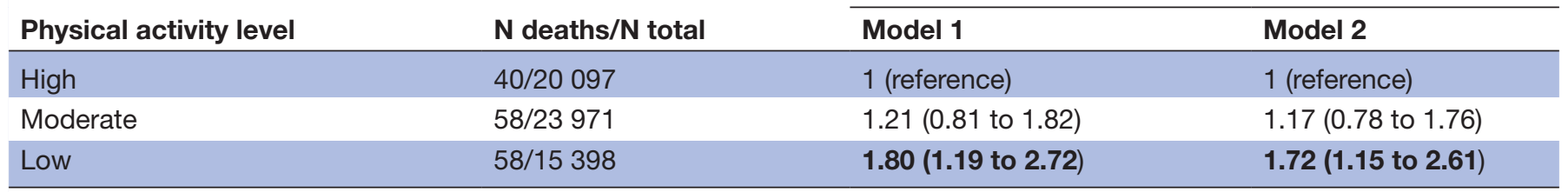

Model 1: adjusted for baseline age, sex, ethnicity, Townsend Deprivation Index, smoking status, alcohol intake, educational attainment, number of illnesses and number of treatments/medications taken.

Model 2: model 1 plus biomarkers associated with COVID-19 mortality: neutrophil count and monocyte count (not adjusted for total leucocyte count to prevent overadjustment).

Significant associations $(p<0.05)$ are highlighted in bold.

may restrict our insight into potential mediators of the protective association of physical activity against COVID-19 mortality. Further investigating such mechanisms is key to developing our understanding of how physical activity may modulate COVID-19 pathology and is necessary to facilitate more valid interpretation of findings.

A key strength of this study is that measures were collected at least 10 years before infection, thus ruling out reverse causation (eg, severe COVID-19 causing a reduction in physical activity, or an increase in inflammatory and cardiometabolic markers). Prospective studies are advantageous in this sense, as they can provide valuable insight into factors that may be protective against adverse COVID-19 outcomes before exposure to SARS-CoV-2, unlike studies in infected patients. Another strength of the present study is that the comprehensive information on participant characteristics within the UK Biobank allowed us to adjust for the potential confounding influence of several demographic, behavioural and health/lifestyle factors.

On the other hand, a limitation of this study is that all analysed risk factors were subject to change between baseline assessment and the COVID-19 pandemic. Reductions in physical activity often occur with ageing, ${ }^{31}$ and the results herein regarding physical activity level may therefore be biased towards the null. It also cannot be ignored that many national policies, including the closing of fitness centres and 'stay-at-home' directives may have posed challenges to regular physical activity during the pandemic. ${ }^{32}$ It is therefore possible that our effect estimates may also underestimate the importance of physical activity due to a discord in the physical activity level reported at baseline compared with that at the time of the COVID-19 pandemic. Another limitation is that physical activity level was based on self-report, which is susceptible to bias due to subjectivity and/or the design of the questionnaire. ${ }^{33}$ This may be of particular consideration when comparing individuals with obesity with normal weight individuals, as a higher BMI has been associated with over-reporting of physical activity. ${ }^{34} 35$ If the true volume and/or intensity of physical activity for individuals with obesity classed as being highly active was lower than reported, our findings may underestimate the protective association of a high physical activity level against
COVID-19 mortality in obesity. However, the influence of such bias may be partly negated by using physical activity as a categorical variable, making the misclassification of physical activity to the extent that it would change categories less likely for a given individual. Nevertheless, future research using objectively assessed physical activity to confirm our findings is warranted.

Additionally, our results may have underestimated the prevalence of the included comorbidities in the study population, as only hospital admission data were used to ascertain this, meaning that our results will not have been sensitive to instances where a comorbidity was present but did not warrant hospital admission. It is also important to consider that the prevalence of several of the included comorbidities has been shown to be greater in individuals with obesity, such as cardiovascular diseases, ${ }^{36}$ and that some of these obesity-associated comorbidities have been associated with a higher risk of COVID-19 mortality. ${ }^{37}$ In individuals with obesity, the presence of certain comorbidities could negatively impact physical activity behaviours, potentially resulting in an association between physical inactivity and a higher risk of COVID-19 mortality that is partly driven by a link between an underlying health condition and COVID-19 mortality. In turn, this could lead to an overestimation of the true protective effect of a high physical activity level against COVID-19 mortality in individuals with obesity. To reduce the potential for such bias, future research may benefit from removing individuals living with certain comorbidities from their study population. This was considered unfeasible in the current study however, due to the low number of outcome events (particularly when stratifying across BMI category/physical activity level combinations). Furthermore, the UK Biobank cohort is less socioeconomically deprived and healthier than the general population. ${ }^{38}$ Although our sample is therefore not representative of the wider population and not suited to make inferences on the true prevalence of COVID-19 mortality during the study period, this does not affect the identification of risk factors for diseases. ${ }^{39}$ However, as the cohort is older than the general population, the findings herein should only be interpreted with respect to middle-aged/older adults. 
To conclude, our results provide evidence indicating the potential for a high physical activity level to protect against COVID-19 mortality in individuals with obesity. However, additional research is required to further elucidate the mechanisms through which this may occur, and to confirm the present findings with the removal of potential bias from using self-reported physical activity and potential confounding from the presence of underlying health conditions in physically inactive individuals. Although the worst of the pandemic may have passed in the UK due to the vaccine rollout, COVID-19 is still inflicting considerable detrimental effects globally, and there is always the possibility of new strains emerging that the vaccine may be less protective against. Therefore, based on the current findings, greater efforts from public health authorities to encourage regular physical activity as a protective measure against COVID-19 should be considered, particularly for individuals with obesity.

Contributors MH and NB conceived the idea for the study. MH conducted the statistical analysis and wrote the manuscript. MJR and AT contributed to the statistical methodology. NB, MJR, AT and DJS critically revised the paper for intellectual content and approved the final manuscript. NB is guarantor for the study.

Funding This research was supported by the National Institute for Health Research (NIHR) Leicester Biomedical Research Centre.

Disclaimer The views expressed are those of the authors and not necessarily those of the NHS, the NIHR or the Department of Health.

Competing interests None declared.

Patient consent for publication Not required.

Ethics approval UK Biobank obtained ethical approval from the North West MultiCentre Research Ethics Committee (REC reference: 11/NW/03820). All participants gave written informed consent before enrolment in the study. Direct dissemination of the results to participants is not applicable. This study was performed under UK Biobank application number 70184.

Provenance and peer review Not commissioned; externally peer reviewed.

Data availability statement Data are available on reasonable request. Data may be obtained from a third party and are not publicly available.

Supplemental material This content has been supplied by the author(s). It has not been vetted by BMJ Publishing Group Limited (BMJ) and may not have been peer-reviewed. Any opinions or recommendations discussed are solely those of the author(s) and are not endorsed by BMJ. BMJ disclaims all liability and responsibility arising from any reliance placed on the content. Where the content includes any translated material, BMJ does not warrant the accuracy and reliability of the translations (including but not limited to local regulations, clinical guidelines, terminology, drug names and drug dosages), and is not responsible for any error and/or omissions arising from translation and adaptation or otherwise.

Open access This is an open access article distributed in accordance with the Creative Commons Attribution Non Commercial (CC BY-NC 4.0) license, which permits others to distribute, remix, adapt, build upon this work non-commercially, and license their derivative works on different terms, provided the original work is properly cited, appropriate credit is given, any changes made indicated, and the use is non-commercial. See: http://creativecommons.org/licenses/by-nc/4.0/.

ORCID iDs

Malik Hamrouni http://orcid.org/0000-0002-5523-5435

David J Stensel http://orcid.org/0000-0001-9119-8590

\section{REFERENCES}

1 Prompetchara E, Ketloy C, Palaga T. Immune responses in COVID-19 and potential vaccines: lessons learned from SARS and MERS epidemic. Asian Pac J Allergy Immunol 2020;38:1-9.
2 Huang Y, Lu Y, Huang Y-M, et al. Obesity in patients with COVID-19: a systematic review and meta-analysis. Metabolism 2020;113:154378.

3 Mauvais-Jarvis F. Aging, male sex, obesity, and metabolic inflammation create the perfect storm for COVID-19. Diabetes 2020;69:1857-63.

4 Gleeson M, Bishop NC, Stensel DJ, et al. The anti-inflammatory effects of exercise: mechanisms and implications for the prevention and treatment of disease. Nat Rev Immunol 2011:11:607-15.

5 Woods JA, Hutchinson NT, Powers SK, et al. The COVID-19 pandemic and physical activity. Sports Med Health Sci 2020;2:55-64.

6 da Silveira MP, da Silva Fagundes KK, Bizuti MR, et al. Physical exercise as a tool to help the immune system against COVID-19: an integrative review of the current literature. Clin Exp Med 2021;21:15-28.

7 Wang M, Baker JS, Quan W, et al. A preventive role of exercise across the coronavirus 2 (SARS-CoV-2) pandemic. Front Physiol 2020;11:572718.

8 Sallis R, Young DR, Tartof SY, et al. Physical inactivity is associated with a higher risk for severe COVID-19 outcomes: a study in 48440 adult patients. Br J Sports Med 2021;55:1099-105.

9 Fischer CP, Berntsen A, Perstrup LB, et al. Plasma levels of interleukin-6 and C-reactive protein are associated with physical inactivity independent of obesity. Scand J Med Sci Sports 2007; $17: 580-7$

10 Loprinzi P, Smit E, Lee H, et al. The "Fit but Fat" paradigm addressed using accelerometer-determined physical activity data. N Am J Med Sci 2014;6:295-301.

11 McAuley PA, Chen H, Lee D-C, et al. Physical activity, measures of obesity, and cardiometabolic risk: the multi-ethnic study of atherosclerosis (MESA). J Phys Act Health 2014;11:831-7.

12 Verch M. COVID-19 vaccines: the pandemic will not end overnight. Lancet Microbe 2021;2:e1.

13 Collins R. What makes UK Biobank special? Lancet 2012;379:1173-4.

14 Townsend P, Phillimore P, Beattie A. Health and deprivation. inequality and the North*. Rev Cuba Hig Epidemiol 1997;35:48-50.

15 Craig CL, Marshall AL, Sjöström M, et al. International physical activity questionnaire: 12 -country reliability and validity. Med Sci Sports Exerc 2003:35:1381-95.

16 IPAQ. Guidelines for Data Processing and Analysis of the International Physical Activity Questionnaire (IPAQ)-Short and Long Forms, 2005. Available: https://biobank.ndph.ox.ac.uk/showcase/ ukb/docs/ipaq_analysis.pdf

17 UK Biobank. Blood sample collection, processing and transport, 2011. Available: https://biobank.ctsu.ox.ac.uk/crystal/crystal/docs/ Bloodsample.pdf

18 Hamer M, Yates T, Demakakos P. Television viewing and risk of mortality: exploring the biological plausibility. Atherosclerosis 2017;263:151-5.

19 Mora S, Cook N, Buring JE, et al. Physical activity and reduced risk of cardiovascular events. Circulation 2007;116:2110-8.

20 Armstrong J, Rudkin JK, Allen N, et al. Dynamic linkage of COVID-19 test results between Public Health England's Second Generation Surveillance System and UK Biobank. Microb Genom 2020;6:e000397.

21 Blair SN, Brodney S. Effects of physical inactivity and obesity on morbidity and mortality: current evidence and research issues. Med Sci Sports Exerc 1999;31:S646-62.

22 Anderson JW, Konz EC, Frederich RC, et al. Long-Term weightloss maintenance: a meta-analysis of US studies. Am J Clin Nutr 2001;74:579-84.

23 Kolin DA, Kulm S, Christos PJ, et al. Clinical, regional, and genetic characteristics of Covid-19 patients from UK Biobank. PLoS One 2020;15:e0241264

24 Hojyo S, Uchida M, Tanaka K, et al. How COVID-19 induces cytokine storm with high mortality. Inflamm Regen 2020;40:37.

25 Alon R, Sportiello M, Kozlovski S, et al. Leukocyte trafficking to the lungs and beyond: lessons from influenza for COVID-19. Nat Rev Immunol 2021;21:49-64.

26 Hu B, Huang S, Yin L. The cytokine storm and COVID-19. J Med Virol 2021;93:250-6.

27 Parasher A. COVID-19: current understanding of its pathophysiology, clinical presentation and treatment. Postgrad Med J 2021;97:312-20.

28 Johannsen NM, Priest EL, Dixit VD, et al. Association of white blood cell subfraction concentration with fitness and fatness. Br J Sports Med 2010;44:588-93.

29 Michishita R, Shono N, Inoue T, et al. Effect of exercise therapy on monocyte and neutrophil counts in overweight women. Am J Med Sci 2010;339:152-6. 
30 Willis EA, Shearer JJ, Matthews CE, et al. Association of physical activity and sedentary time with blood cell counts: National health and nutrition survey 2003-2006. PLoS One 2018;13:e0204277.

31 Milanović Z, Pantelić S, Trajković N, et al. Age-Related decrease in physical activity and functional fitness among elderly men and women. Clin Interv Aging 2013;8:549-56.

32 Hall G, Laddu DR, Phillips SA, et al. A tale of two pandemics: how will COVID-19 and global trends in physical inactivity and sedentary behavior affect one another? Prog Cardiovasc Dis 2021;64:108-10.

33 Olds TS, Gomersall SR, Olds ST, et al. A source of systematic bias in self-reported physical activity: the cutpoint bias hypothesis. J Sci Med Sport 2019;22:924-8.

34 Slootmaker SM, Schuit AJ, Chinapaw MJ, et al. Disagreement in physical activity assessed by accelerometer and self-report in subgroups of age, gender, education and weight status. Int J Behav Nutr Phys Act 2009;6:17.

35 Pietiläinen $\mathrm{KH}$, Korkeila M, Bogl LH, et al. Inaccuracies in food and physical activity diaries of obese subjects: complementary evidence from doubly labeled water and co-twin assessments. Int $J$ Obes 2010;34:437-45

36 Lavie CJ, Milani RV, Ventura HO. Obesity and cardiovascular disease: risk factor, paradox, and impact of weight loss. J Am Coll Cardiol 2009;53:1925-32.

37 de Almeida-Pititto B, Dualib PM, Zajdenverg L, et al. Severity and mortality of COVID 19 in patients with diabetes, hypertension and cardiovascular disease: a meta-analysis. Diabetol Metab Syndr 2020;12:75.

38 Fry A, Littlejohns TJ, Sudlow C, et al. Comparison of sociodemographic and health-related characteristics of UK Biobank participants with those of the general population. Am J Epidemiol 2017:186:1026-34.

39 Batty GD, Gale CR, Kivimäki M, et al. Comparison of risk factor associations in UK Biobank against representative, general population based studies with conventional response rates: prospective cohort study and individual participant meta-analysis. BMJ 2020;368:m131. 